\title{
Clinical-Pathological Features and Treatment Outcome of Patients With Hobnail Variant Papillary Thyroid Carcinoma
}

\author{
Anello Marcello Poma ${ }^{1}$, Elisabetta Macerola ${ }^{1}$, Agnese Proietti ${ }^{1}$, Paola Vignali ${ }^{1}$, \\ Rebecca Sparavelli ${ }^{1}$, Liborio Torregrossa ${ }^{1}$, Antonio Matrone ${ }^{2}$, Alessio Basolo ${ }^{2}$, \\ Rossella Elisei ${ }^{2}$, Ferruccio Santini ${ }^{2}$ and Clara Ugolini ${ }^{1 *}$ \\ 1 Department of Surgical, Medical, Molecular Pathology and Critical Area, University of Pisa, Pisa, Italy, \\ 2 Department of Clinical and Experimental Medicine, University of Pisa, Pisa, Italy
}

OPEN ACCESS

Edited by:

Marialuisa Appetecchia, Hospital Physiotherapy Institutes

(IRCCS), Italy

Reviewed by:

Salvatore Monti,

Azienda Ospedaliera Sant'

Andrea, Italy

Laura Deborah Locati,

National Cancer Institute Foundation

(IRCCS), Italy

*Correspondence:

Clara Ugolini

clara.ugolini@gmail.com

Specialty section:

This article was submitted to

Cancer Endocrinology,

a section of the journal

Frontiers in Endocrinology

Received: 23 December 2021

Accepted: 07 February 2022

Published: 02 March 2022

Citation:

Poma AM, Macerola E, Proietti A

Vignali $P$, Sparavelli $R$, Torregrossa $L$,

Matrone A, Basolo A, Elisei R, Santini F

and Ugolini C (2022) Clinical-

Pathological Features and Treatment

Outcome of Patients With Hobnail

Variant Papillary Thyroid Carcinoma.

Front. Endocrinol. 13:842424.

doi: 10.3389/fendo.2022.842424
Papillary thyroid carcinoma (PTC) with hobnail areas above $30 \%$ is classified as hobnail variant (HVPTC). Although it is widely accepted that HVPTC has a worse outcome than classical PTC, it is unclear whether PTC with hobnail features below $30 \%$ is as aggressive as HVPTC. We gathered the largest mono-institutional series of PTC with hobnail areas and HVPTC to evaluate differences in terms of pathological features of aggressiveness, molecular profile, and treatment outcome. A total of 99 PTC with hobnail features above $5 \%$ were retrospectively selected; 34 of them met the criteria for HVPTC $(0.4 \%$ of all PTC diagnosed at our institution). All tumors showed high rates of extra-thyroidal extension (40.4\%), lymph node metastasis (68.1\% of patients with lymphadenectomy), and vascular emboli (49.5\%), with no differences according to the 30\% cutoff. On the other hand, distant metastases were present in HVPTC only (9.4\%). Also, advanced age, advanced disease stage, and TERT promoter mutation were associated with HVPTC. More than half of the patients with follow-up had structural or biochemical persistence after 1 year from surgery. Structural persistence was significantly more common in patients with HVPTC (37.5\% vs. $8.7 \%)$, while no differences were observed considering structural and biochemical persistence together. The presence of hobnail features identifies locally aggressive tumors, and, consequently, it should be always acknowledged in the pathological report. However, tumors with more than 30\% hobnail areas frequently present TERT promoter mutations, advanced disease stage, and structural persistence after radioiodine ablation.

Keywords: hobnail variant, papillary thyroid carcinoma, PTC, treatment outcome, BRAF, TERT, RET/PTC

\section{INTRODUCTION}

Thyroid carcinoma is the most common endocrine malignancy. The great majority of them are well-differentiated carcinomas, with papillary thyroid carcinoma (PTC) being the most common histotype (1). PTC has generally an excellent outcome following the traditional treatments (i.e., surgery with or without radioiodine ablation) (2). However, some PTC variants are considered more 
aggressive due to high rates of pathological features of invasion and a troubled clinical management. Aggressive PTC variants include tall cell, columnar, diffuse sclerosing, solid, and hobnail variants $(3,4)$.

The hobnail variant of papillary thyroid carcinoma (HVPTC) was firstly described by Kakudo et al. in 2004, which noted loss of cell polarity, high nuclear/cytoplasmic ratio, and apical nuclear position that produce a surface bulge and confer the cell a hobnail appearance. The authors attributed these morphologic changes to poor cellular differentiation (5). This suspicion was confirmed by a case series from the Mayo Clinic in 2010, which further described HVPTC and confirmed an aggressive clinical behavior including higher rates of distant metastases, radioiodine refractoriness, and mortality compared with classical variant PTC (CVPTC) (6). Subsequently, other authors have confirmed the poorer outcome of HVPTC patients compared to CVPTC (7-11). Since the latest World Health Organization (WHO) classification of endocrine tumors defines HVPTC by the presence of at least $30 \%$ of cells with hobnail features (1), it is not clear whether tumors with lower proportions of hobnail areas deserve to be classified as HVPTC. Some authors have reported that patients with PTC presenting $10 \%$ to $30 \%$ of hobnail features have similar rates of aggressive pathological features and outcome to that of HVPTC patients $(9,12)$.

From a molecular point of view, HVPTC presents a very high $B R A F$ V600E prevalence (i.e., up to $80 \%$ ), followed by RET/PTC rearrangements. Secondary mutations were also described, especially in TP53 and TERT promoter $(1,6,8,12,13)$.

Since HVPTC is a rare entity, accounting for approximately $1 \%$ of all PTC, the case series presented in literature included a limited number of tumors (14). Larger series are then required to answer the still open questions, especially related to tumors with hobnail features lower than $30 \%$.

Herein, we reported the largest mono-institutional series of PTC with hobnail features above $5 \%$ by retrospectively reviewing cases with hobnail areas over the last 6 years. We aimed at investigating differences in terms of clinical-pathological, molecular characteristics, and treatment outcome between HVPTC (i.e., 30\% or more hobnail features) and PTC with less than $30 \%$ hobnail areas.

\section{MATERIALS AND METHODS}

\section{Study Cohort}

A retrospective search was conducted in the institutional database by including all the histological reports of PTC containing the term "hobnail" in the period 2015-2020. For each of the retrieved reports, the entire series of hematoxylin and eosin slides were carefully reviewed by three expert pathologists (CU, AgP, and LT), who independently determined the percentage of hobnail areas. The median value was used as final percentage unless a disagreement of $20 \%$ or more was present. In these cases, slides were collegially discussed until mutual agreement. Clinical-pathological characteristics of tumors were also collected. A minimal cutoff of 5\% hobnail areas was set. Hence, all PTC with at least 5\% hobnail areas diagnosed between January 2015 and December 2020 at our institution were included in the study. Cases with foci of poorly differentiated or anaplastic thyroid carcinomas were excluded. All cases were re-staged according to the latest edition of the AJCC/TNM classification of thyroid tumors (15).

In a subset of patients, treatment data including type of surgery and radioiodine ablation were available. Patients were evaluated during the follow-up with regular clinical, biochemical, and imaging procedures, according to the standard of care. At last evaluation, response to the treatment was defined according to the 2015 ATA guidelines (2).

The study fulfills the standards of the Declaration of Helsinki and its subsequent amendments, and was approved by the ethics committee. Written informed consent was signed by each patient.

\section{Molecular Analyses}

For each case, one paraffin block was selected, and four $10-\mu \mathrm{m}$ thick sections of tissue were obtained for nucleic acids extraction. After standard deparaffination, tissue enrichment was performed by manually dissecting the areas containing tumor cells. The Qiamp DNA Mini kit and the RNeasy FFPE kit were used for DNA and RNA extraction, respectively (Qiagen, Hilden, Germany).

The presence of mutations in BRAF exon 15 and in the promoter of TERT was investigated by PCR followed by direct sequencing, as described previously (16). The analysis of RET/ $P T C$ fusions was conducted in all the $B R A F$-negative cases by using a one-step reverse transcriptional PCR kit, the EasyPGX Ready Thyroid Fusion (Diatech Pharmacogenetics, Jesi, AN, Italy).

\section{Statistics}

Continuous variables were tested for normality distribution by the Shapiro-Wilk test. Variables that did not follow a normal distribution are presented as median and interquartile range (IQR), and were tested by the Mann-Whitney $U$ test. Normally distributed variables are presented as mean and standard deviation, and were tested by the Welch $t$-test. For categorical variables, the Chi-square test with Yates' correction was used; the Fisher exact test was run whenever appropriate. In multivariate setting, a logistic regression was performed, and the Box-Tidwell test was used to check the linear relationship between continuous variables used in the model and the log odds of the outcome. A cutoff of $p=0.05$ was set as significance level. All analyses were performed in R environment (https://www.r-project.org/, v 4.1.1, last accessed Dec 15, 2021).

\section{RESULTS}

\section{Clinical-Pathological and Molecular Features}

From 2015 to 2020, a total of 9,162 PTC were diagnosed at our institution. Among them, 99 PTC (1.1\%) had hobnail areas and were included in the study (Figure 1). Thirty-four PTC with hobnail areas ( $0.4 \%$ of total PTC) met the criteria of HVPTC (i.e., at least $30 \%$ of hobnail areas), while 65 cases were PTC with hobnail areas between $5 \%$ and $25 \%$, including 45 classic variants 
(CVPTC), 15 tall cell variants (TCPTC), 4 solid-trabecular variants (TSVPTC), 1 clear cell variant (CCVPTC).

Overall, the mean age was $49.8( \pm 15.9)$ years, the median tumor size was $1.8 \mathrm{~cm}$ (IQR 1.3-2.4), and the female-to-male ratio was 1.4. Only 3 PTC (3\%) were encapsulated non-invasive. On the contrary, aggressive features were often present, including $40.4 \%$ of extra-thyroidal extension (ETE, $6 \%$ with T3b or T4 disease), $49.5 \%$ of vascular invasion, and $32.3 \%$ of lymph node metastases (68.1\% of those with lymphadenectomy). $B R A F$ mutation (V600E) was present in 69 out of $88(78.4 \%)$ analyzed cases. Six tumors (6.8\%) harbored a RET/PTC rearrangement, including $4 R E T / P T C 1$ and 2 RET/PTC3. TERT promoter mutation was successfully tested in 53 cases, and 8 of them (15.1\%) presented the C228T mutation.

Patients with HVPTC were older than those with PTC with hobnail features $(p=0.002)$ and presented more often bilateral tumors $(p=0.02)$ and distant metastases at diagnosis $(p=0.03)$. In addition, HVPTC tumors presented more frequently at advanced stages $(p=0.05)$ and were associated with TERT promoter mutation $(p=0.02)$. BRAF mutations and RET fusions were not associated with hobnail percentage $(p=1$ and $p=0.66$ respectively).

Detailed clinical-pathological and mutational data are reported in Table $\mathbf{1}$.

\section{Treatment and Patients' Outcome}

All patients underwent total thyroidectomy, and lymph node dissection was performed on 47 of them (47.5\%). According to the latest ATA risk stratification system (2), 17 patients were low risk $(17.7 \%), 68$ were intermediate risk $(70.8 \%)$, and 11 were high risk $(11.5 \%)$. In three cases, there were no sufficient data to determine the ATA risk category. As expected, PTC with hobnail features were associated with ATA low risk $(p=0.005)$ since patients with HVPTC cannot be classified as low risk (2). For a subset of patients $(n=39)$, follow-up data were available including 16 HVPTC and 23 PTC with hobnail features. The median follow-up was 1 year after surgery. Thirty-three patients $(84.6 \%)$ received radioiodine ablation, and three of them $(7.7 \%$ of patients with follow-up) also underwent external beam radiation therapy. No differences between HVPTC and PTC with hobnail features were observed in terms of rate of lymphadenectomy and radioiodine ablation $(68.7 \%$ vs. $60.9 \%, p=0.74$ and $81.2 \%$ vs. $86.9 \%, p=0.67$, respectively). Among patients with follow-up data, $19(48.7 \%)$ had excellent response to therapy (i.e., no clinical, biochemical or structural evidence of disease), eight (20.5\%) had structural incomplete response, and 12 (30.8\%) had indeterminate response (measurable anti-Tg antibody levels). Structural persistence was associated with HVPTC patients $(37.5 \%$ vs. $8.7 \%, p=0.04)$, while no differences were observed when considering structural or biochemical persistence together $(62.5 \%$ vs. $43.5 \%, p=0.40)$.

We tried to find predictors of structural persistence by a multivariate logistic regression model considering PTC variant (i.e., HVPTC vs. PTC with hobnail features) and the identified confounders (i.e., age, bilaterality, and TERT promoter mutation). No strong predictors of structural recurrence were

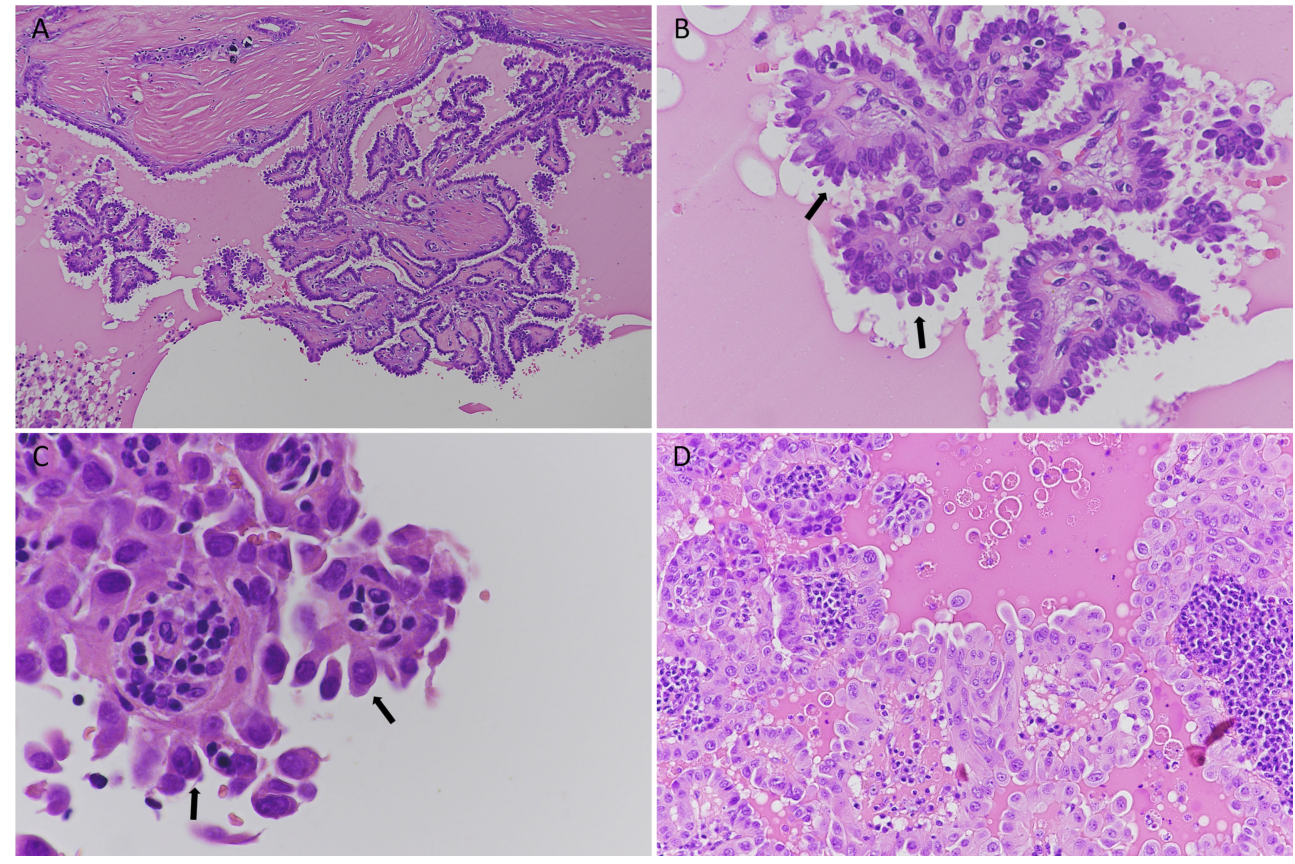

FIGURE 1 | Histological features of hobnail variant papillary thyroid carcinoma (HVPTC); hematoxylin and eosin staining. (A-C) A case of HVPTC: (A) low magnification (original magnification 10x); intermixed papillary structures lined by neoplastic cells; (B) 40x magnification of papillary structures. Hobnail cells are evident (black arrows); (C) details of nuclear atypia in hobnail-shaped cells (black arrows, 60x); (D) a case of papillary thyroid carcinoma with hobnail features (20x). 
TABLE 1 | Clinical-pathological and molecular characteristics of HVPTC and PTC with hobnail features.

\begin{tabular}{|c|c|c|c|c|}
\hline \multicolumn{2}{|c|}{ Pathological and molecular features } & \multirow{2}{*}{$\begin{array}{c}\text { HVPTC }(\boldsymbol{n}=\mathbf{3 4})^{\S} \\
13(38.2 \%)\end{array}$} & \multirow{2}{*}{$\begin{array}{c}\text { PTC with hobnail features }(\boldsymbol{n}=65)^{\S} \S \\
28(43.1 \%)\end{array}$} & \multirow{2}{*}{$\frac{\boldsymbol{p} \text {-value }}{0.80}$} \\
\hline Gender & male & & & \\
\hline Age & years, mean (SD) & $56.4(13.5)$ & $46.3(16.1)$ & 0.002 \\
\hline Size & cm, median (IQR) & $1.8(1.0-2.6)$ & $1.8(1.3-2.4)$ & 0.93 \\
\hline \multirow[t]{2}{*}{ Extra-thyroidal extension } & minimal & $8(25.0 \%)$ & $26(40.6 \%)$ & 0.31 \\
\hline & gross & $3(9.4 \%)$ & $3(4.7 \%)$ & \\
\hline \multirow[t]{2}{*}{ Number of emboli } & $<4$ & $3(9.4 \%)$ & $9(14.1 \%)$ & 0.87 \\
\hline & $\geq 4$ & $11(34.9 \%)$ & $24(37.5 \%)$ & \\
\hline Multifocal tumor & & $18(56.2 \%)$ & $27(42.2 \%)$ & 0.28 \\
\hline Bilateral tumor & & $16(50.0 \%)$ & $15(23.4 \%)$ & 0.02 \\
\hline Thyroiditis & & $15(46.9 \%)$ & $18(28.1 \%)$ & 0.11 \\
\hline \multirow[t]{3}{*}{ Pathologic T stage } & $\mathrm{T} 1$ & $21(65.6 \%)$ & 39 (60.9\%) & 0.12 \\
\hline & $\mathrm{T} 2$ & $5(15.6 \%)$ & $20(31.2 \%)$ & \\
\hline & Т3-4 & $6(18.7 \%)$ & $5(7.8 \%)$ & \\
\hline \multirow[t]{2}{*}{ Pathologic N stage* } & $\mathrm{N} 1 \mathrm{a}$ & $5(38.5 \%)$ & $12(35.3 \%)$ & 1 \\
\hline & N1b & $4(30.8 \%)$ & $11(32.3 \%)$ & \\
\hline Extra-nodal extension ${ }^{\star \star}$ & & 3 (33.3\%) & $4(17.4 \%)$ & 0.37 \\
\hline Distant metastases at diagnosis & & $3(9.4 \%)$ & 0 & 0.03 \\
\hline \multirow[t]{3}{*}{ AJCC TNM stage } & stage I & $24(75 \%)$ & 59 (92.2\%) & 0.05 \\
\hline & stage ॥ & $5(15.6 \%)$ & $3(4.7 \%)$ & \\
\hline & stage III-IV & $3(9.4 \%)$ & $2(3.1 \%)$ & \\
\hline BRAF mutation & & $24(77.4 \%)$ & 45 (78.9\%) & 1 \\
\hline RET/PTC rearrangement & & $1(3.2 \%)$ & $5(8.8 \%)$ & 0.66 \\
\hline TERT promoter mutation & & 7 (30.4\%) & $1(3.3 \%)$ & 0.02 \\
\hline Lymphadenectomy ${ }^{\S \S \S}$ & & $11(68.7 \%)$ & $14(60.9 \%)$ & 0.74 \\
\hline RAl ablation $\$ \S \S$ & & $13(81.2 \%)$ & 20 (86.9\%) & 0.67 \\
\hline Structural persistence ${ }^{\S \S \S}$ & & $6(37.5 \%)$ & $2(8.7 \%)$ & 0.04 \\
\hline Biochemical persistence $e^{\S \S}$ & & $4(25.0 \%)$ & 8 (34.8\%) & 0.73 \\
\hline
\end{tabular}

HVPTC, hobnail variant papillary thyroid carcinoma; PTC, papillary thyroid carcinoma; SD, standard deviation; IQR, interquartile range; AJCC, American Joint Committee on Cancer; TNM, tumor node metastases; RAl, radioactive iodine.

$\$ 2$ patients with data not available.

$\$ \$ 1$ patient with data not available.

\$\$\$Cases with follow-up data (i.e., 16 HVPTC and 23 PTC with hobnail features).

*Only cases with lymphadenectomy were considered.

** Only cases with lymph node metastases were considered.

In bold are significant $p$-values.

identified, but a trend was observed for HVPTC $(p=0.09)$ and TERT promoter mutation $(p=0.10)$. The complete results of multivariate analysis are reported in Table 2.

\section{DISCUSSION}

The hobnail variant papillary thyroid carcinoma (HVPTC) was described for the first time in 2004 (5), and it was referenced in the WHO classification of endocrine tumors only in 2017 (1). Since the first reports, HVPTC was recognized as an aggressive variant $(5,6)$, and nowadays it is widely accepted that patients with HVPTC have a worse outcome than those with CVPTC (6$11,17)$. However, owing to its rarity, there is a need for a wider characterization of tumors with hobnail features, both molecularly and clinically. In particular, the 30\% diagnostic cutoff represents a matter of discussion. Tumors with $10 \%$ to $30 \%$ hobnail features have been associated with poor outcome $(9$, $12,14)$, and a refinement of the diagnostic criteria was already proposed (14). In the present study, we retrospectively gathered the largest series of HVPTC and PTC with 5\% to $30 \%$ of hobnail areas. HVPTC and PTC with hobnail features did not show evident

TABLE 2 | Multivariate logistic regression model to identify predictors of structural persistence.

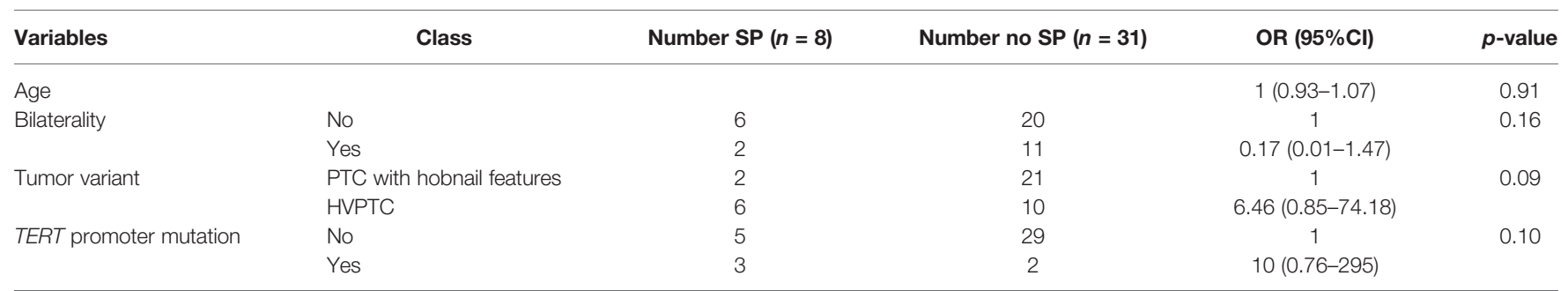

SP, structural persistence; OR, odds ratio; Cl, confidence interval; PTC, papillary thyroid carcinoma; HVPTC, hobnail variant papillary thyroid carcinoma. 
differences in pathological features of local aggressiveness (i.e., tumor size, extra-thyroidal extension, lymph node metastasis, and vascular emboli). However, distant metastases at diagnosis were present in HVPTC patients only. The latest American Joint Committee on Cancer-tumor node metastasis (AJCC-TNM) staging system determined a remarkable downstaging of thyroid tumors. Accordingly, less than 5\% of patients with differentiated thyroid cancer (DTC) should be diagnosed at stage III-IV (18-20). In our series, despite the highly prevalent pathological aggressive features, more than $90 \%$ of PTC with hobnail features were diagnosed at stage I, while up to $25 \%$ of HVPTC were at disease stage II or higher, including almost $10 \%$ of stage III or IV. Therefore, our results confirm that HVPTC patients are expected to have a worse prognosis than those with DTC. It has to be pointed out that the disease stage imbalances herein observed may be due, at least in part, to the older age of patients with HVPTC. Nevertheless, the prevalence of TERT promoter mutation observed in HVPTC (i.e., $30 \%$ ) is in the middle between DTC and ATC, and may reflect a halfway survival rate as well (21). The response to treatment outcome obtained in our series may offer additional perspectives. In our study, although patients underwent similar treatment, structural persistence was more common in those with HVPTC (37.5\% vs. $8.7 \%$ in PTC with hobnail features), while no significant differences were observed in terms of structural or biochemical persistence considered together $(62.5 \%$ vs. $43.5 \%)$. It was highlighted that in patients with biochemical incomplete response, only a relatively small proportion actually develop structural evidence of disease (22-24), even though subgroups of patients may have a higher risk $(25,26)$. In addition, the $8.7 \%$ of structural persistence observed in PTC with hobnail features is in line with the $7.2 \%$ observed in our previous study on CVPTC (27).

This study suffers from some limitations. First, since our institution is a national referral center, many patients that undergo surgery are lost during the follow-up; consequently, the follow-up is short (i.e., approximately 1 year after surgery) and available in a limited number of cases. Hence, we could not evaluate disease recurrence/progression or survival. Second, the sample size is limited, especially for multivariate analysis; as a consequence, the $95 \%$ CI of regression analysis is exceptionally wide. On the other hand, this is the largest mono-institutional series of HVPTC and PTC with hobnail features; thus, bias due to subjective morphological interpretation is reduced.

\section{REFERENCES}

1. Lloyd R, Osamura R, Klöppel G, Rosai J. WHO Classification of Tumours of Endocrine Organs. 4th ed. Lyon: IARC press (2017).

2. Haugen BR, Alexander EK, Bible KC, Doherty GM, Mandel SJ, Nikiforov YE, et al. 2015 American Thyroid Association Management Guidelines for Adult Patients With Thyroid Nodules and Differentiated Thyroid Cancer: The American Thyroid Association Guidelines Task Force on Thyroid Nodules and Differentiated Thyroid Cancer. Thyroid (2016) 26:1-133. doi: 10.1089/ thy. 2015.0020

3. Baloch ZW, LiVolsi VA. Special Types of Thyroid Carcinoma. Histopathology (2018) 72:40-52. doi: 10.1111/his.13348

4. Nath MC, Erickson LA. Aggressive Variants of Papillary Thyroid Carcinoma: Hobnail, Tall Cell, Columnar, and Solid. Adv Anat Pathol (2018) 25:172-9. doi: 10.1097/PAP.0000000000000184
In conclusion, we demonstrated that HVPTC and PTC with hobnail areas showed high rates of locally aggressive features and a relatively low rate of excellent response after standard therapy. However, HVPTC also presented high-risk features such as advanced age, TERT promoter mutation, distant metastases, and advanced stage disease. In addition, patients with HVPTC had a high rate of structural persistent disease after radioiodine ablation, which was much higher than in patients with PTC with hobnail areas below 30\%. Long-term follow-up data in large monocentric studies will establish whether PTC with hobnail features should be considered as high-risk tumors as well. The presence of any proportion of hobnail features in PTCs should be at least acknowledged in the pathological report.

\section{DATA AVAILABILITY STATEMENT}

The original contributions presented in the study are included in the article/supplementary material. Further inquiries can be directed to the corresponding author.

\section{ETHICS STATEMENT}

The studies involving human participants were reviewed and approved by CEAVNO. The patients/participants provided their written informed consent to participate in this study.

\section{AUTHOR CONTRIBUTIONS}

Conceptualization: AnP, RE, FS, and CU. Methodology: AnP, EM, AgP, PV, RS, LT, AM, AB, and CU. Formal analysis: AnP, EM, and CU. Writing-original draft preparation: AnP, EM, and CU. Writing-review and editing: all authors. Supervision, RE, FS, and CU. All authors contributed to the article and approved the submitted version.

\section{FUNDING}

This study was funded by the University of Pisa (no specific grant).

5. Kakudo K, Tang W, Ito Y, Mori I, Nakamura Y, Miyauchi A. Papillary Carcinoma of the Thyroid in Japan: Subclassification of Common Type and Identification of Low Risk Group. J Clin Pathol (2004) 57:1041-6. doi: $10.1136 /$ jcp.2004.017889

6. Asioli S, Erickson LA, Sebo TJ, Zhang J, Jin L, Thompson GB, et al. Papillary Thyroid Carcinoma With Prominent Hobnail Features: A New Aggressive Variant of Moderately Differentiated Papillary Carcinoma. A Clinicopathologic, Immunohistochemical, and Molecular Study of Eight Cases. Am J Surg Pathol (2010) 34:44-52. doi: 10.1097/PAS.0b013e3181c46677

7. Amacher AM, Goyal B, Lewis JS, El-Mofty SK, Chernock RD. Prevalence of a Hobnail Pattern in Papillary, Poorly Differentiated, and Anaplastic Thyroid Carcinoma: A Possible Manifestation of High-Grade Transformation. Am J Surg Pathol (2015) 39:260-5. doi: 10.1097/PAS.0000000000000329

8. Lubitz CC, Economopoulos KP, Pawlak AC, Lynch K, Dias-Santagata D, Faquin WC, et al. Hobnail Variant of Papillary Thyroid Carcinoma: An 
Institutional Case Series and Molecular Profile. Thyroid (2014) 24:958-65. doi: $10.1089 /$ thy.2013.0573

9. Asioli S, Erickson LA, Righi A, Lloyd RV. Papillary Thyroid Carcinoma With Hobnail Features: Histopathologic Criteria to Predict Aggressive Behavior. Hum Pathol (2013) 44:320-8. doi: 10.1016/j.humpath.2012.06.003

10. Asioli S, Maletta F, Pagni F, Pacchioni D, Vanzati A, Mariani S, et al. Cytomorphologic and Molecular Features of Hobnail Variant of Papillary Thyroid Carcinoma: Case Series and Literature Review. Diagn Cytopathol (2014) 42:78-84. doi: 10.1002/dc.23028

11. Morandi L, Righi A, Maletta F, Rucci P, Pagni F, Gallo M, et al. Somatic Mutation Profiling of Hobnail Variant of Papillary Thyroid Carcinoma. Endocr Relat Cancer (2017) 24:107-17. doi: 10.1530/ERC-16-0546

12. Watutantrige-Fernando S, Vianello F, Barollo S, Bertazza L, Galuppini F, Cavedon E, et al. The Hobnail Variant of Papillary Thyroid Carcinoma: Clinical/Molecular Characteristics of a Large Monocentric Series and Comparison With Conventional Histotypes. Thyroid (2018) 28:96-103. doi: 10.1089 /thy.2017.0248

13. Teng L, Deng W, Lu J, Zhang J, Ren X, Duan H, et al. Hobnail Variant of Papillary Thyroid Carcinoma: Molecular Profiling and Comparison to Classical Papillary Thyroid Carcinoma, Poorly Differentiated Thyroid Carcinoma and Anaplastic Thyroid Carcinoma. Oncotarget (2017) 8:2202333. doi: 10.18632 /oncotarget.15786

14. Donaldson LB, Yan F, Morgan PF, Kaczmar JM, Fernandes JK, Nguyen SA, et al. Hobnail Variant of Papillary Thyroid Carcinoma: A Systematic Review and Meta-Analysis. Endocrine (2021) 72:27-39. doi: 10.1007/s12020-02002505-z

15. Tuttle M, Morris LF, Haugen B, Shah J, Sosa JA, Rohren E, et al. ThyroidDifferentiated and Anaplastic Carcinoma (Chapter 73). In: MB Amin, SB Edge, F Greene, D Byrd, RK Brookland, MK Washington, JE Gershenwald, CC Compton, KR Hess, DC Sullivan, JM Jessup, J Brierley, LE Gaspar, RL Schilsky, CM Balch, DP Winchester, EA Asare, M Madera, DM Gress, LR Meyer, editors. AJCC Cancer Staging Manual, 8th ed. New York City: Springer International Publishing (2017).

16. Macerola E, Loggini B, Giannini R, Garavello G, Giordano M, Proietti A, et al. Coexistence of TERT Promoter and BRAF Mutations in Cutaneous Melanoma is Associated With More Clinicopathological Features of Aggressiveness. Virchows Arch (2015) 467:177-84. doi: 10.1007/s00428-0151784-x

17. Lee YS, Kim Y, Jeon S, Bae JS, Jung SL, Jung CK. Cytologic, Clinicopathologic, and Molecular Features of Papillary Thyroid Carcinoma With Prominent Hobnail Features: 10 Case Reports and Systematic Literature Review. Int J Clin Exp Pathol (2015) 8:7988-97.

18. Tam S, Boonsripitayanon M, Amit M, Fellman BM, Li Y, Busaidy NL, et al. Survival in Differentiated Thyroid Cancer: Comparing the AJCC Cancer Staging Seventh and Eighth Editions. Thyroid (2018) 28:1301-10. doi: 10.1089 /thy.2017.0572

19. Lamartina L, Grani G, Arvat E, Nervo A, Zatelli MC, Rossi R, et al. 8th Edition of the AJCC/TNM Staging System of Thyroid Cancer: What to Expect (ITCO\#2). Endocr Relat Cancer (2018) 25:L7-11. doi: 10.1530/ERC-17-0453
20. Pontius LN, Oyekunle TO, Thomas SM, Stang MT, Scheri RP, Roman SA, et al. Projecting Survival in Papillary Thyroid Cancer: A Comparison of the Seventh and Eighth Editions of the American Joint Commission on Cancer/ Union for International Cancer Control Staging Systems in Two Contemporary National Patient Cohorts. Thyroid (2017) 27:1408-16. doi: 10.1089/thy.2017.0306

21. Jin $\mathrm{A}, \mathrm{Xu}$ J, Wang Y. The Role of TERT Promoter Mutations in Postoperative and Preoperative Diagnosis and Prognosis in Thyroid Cancer. Med (Baltimore) (2018) 97:e11548. doi: 10.1097/MD.0000000000011548

22. Pitoia F, Fabián P, Abelleira E, Erika A, Tala H, Hernán T, et al. Biochemical Persistence in Thyroid Cancer: Is There Anything to Worry About? Endocrine (2014) 46:532-7. doi: 10.1007/s12020-013-0097-6

23. Tuttle RM. Optimal Management of a Biochemical Incomplete Response to Therapy in Differentiated Thyroid Cancer: Aggressive Treatment or Cautious Observation? Endocrine (2014) 46:363-4. doi: 10.1007/s12020-014-0213-2

24. Zern NK, Clifton-Bligh R, Gill AJ, Aniss A, Sidhu S, Delbridge L, et al. Disease Progression in Papillary Thyroid Cancer With Biochemical Incomplete Response to Initial Therapy. Ann Surg Oncol (2017) 24:2611-6. doi: 10.1245/s10434-017-5911-6

25. Steinschneider M, Pitaro J, Koren S, Mizrakli Y, Benbassat C, Muallem Kalmovich L. Differentiated Thyroid Cancer With Biochemical Incomplete Response: Clinico-Pathological Characteristics and Long Term Disease Outcomes. Cancers (Basel) (2021) 13:5422. doi: 10.3390/cancers13215422

26. Ahn J, Song E, Kim WG, Kim TY, Kim WB, Shong YK, et al. Long-Term Clinical Outcomes of Papillary Thyroid Carcinoma Patients With Biochemical Incomplete Response. Endocrine (2020) 67:623-9. doi: 10.1007/ s12020-019-02142-1

27. Giani C, Torregrossa L, Piaggi P, Matrone A, Viola D, Molinaro E, et al. Outcome of Classical (CVPTC) and Follicular (FVPTC) Variants of Papillary Thyroid Cancer: 15 Years of Follow-Up. Endocrine (2020) 68:607-16. doi: $10.1007 / \mathrm{s} 12020-020-02229-0$

Conflict of Interest: The authors declare that the research was conducted in the absence of any commercial or financial relationships that could be construed as a potential conflict of interest.

Publisher's Note: All claims expressed in this article are solely those of the authors and do not necessarily represent those of their affiliated organizations, or those of the publisher, the editors and the reviewers. Any product that may be evaluated in this article, or claim that may be made by its manufacturer, is not guaranteed or endorsed by the publisher.

Copyright (c) 2022 Poma, Macerola, Proietti, Vignali, Sparavelli, Torregrossa, Matrone, Basolo, Elisei, Santini and Ugolini. This is an open-access article distributed under the terms of the Creative Commons Attribution License (CC BY). The use, distribution or reproduction in other forums is permitted, provided the original author(s) and the copyright owner(s) are credited and that the original publication in this journal is cited, in accordance with accepted academic practice. No use, distribution or reproduction is permitted which does not comply with these terms. 